\title{
Including PML-Based Absorbing Boundary Conditions in the MLFMA
}

\author{
Davy Pissoort, Dries Vande Ginste, and Frank Olyslager, Fellow, IEEE
}

\begin{abstract}
In this letter, the multilevel fast multipole algorithm is extended to the use of complex coordinates. These complex coordinates appear, e.g., when extracting the $S$-parameters of an electromagnetic crystal device terminated by perfectly matched layer based absorbing boundary conditions with the multiple scattering technique. The coordinates of the centers of the boxes on the different levels in the multilevel multipole algorithm are chosen so that they follow the path according to which the coordinates of the cylinders' centers become complex. Therefore, a new real coordinate is introduced along this path. The boxes are first constructed based on this real coordinate and after that the corresponding complex coordinates are calculated. The described scheme is applied to the analysis of a multiplexer-demultiplexer device.
\end{abstract}

Index Terms-Electromagnetic crystals, fast multipole methods, perfectly matched layers (PMLs), photonic crystals.

\section{INTRODUCTION}

$\mathbf{R}$ ECENTLY, a new perfectly matched layer (PML)-based absorbing boundary condition was introduced for the termination of periodic waveguides in integral equation like simulation techniques. In [1], this PML-based absorbing boundary condition was applied to the characterization of two-dimensional (2-D) electromagnetic crystal (EC) devices with the multiple scattering technique (MST) [2], [3]. Two-dimensional (2-D) ECs consist of parallel homogeneous dielectric cylinders residing on a periodic lattice in a homogeneous background medium. The underlying periodic structure of an EC gives rise to the appearance of frequency ranges (electromagnetic bandgaps) for which the electromagnetic fields cannot propagate inside the crystal. Disruption of the crystal periodicity by introducing crystal defects induces a field localization that allows the design of various interesting devices.

Most often, the goal of the simulations is the knowledge of the $S$-parameters of the EC device. Therefore, it is advantageous to be able to model EC waveguide appendages that are infinitely long. Exploiting the complex coordinate interpretation of a PML [4], [5], the EC waveguides that constitute the ports of the EC device are terminated by adding a couple for which the cylinders have complex coordinates. If the waveguide enters the complex plane in a smooth way, reflections caused by the periodicity disturbance are low and at the same time a significant absorption is achieved.

The main disadvantage of an integral equation technique is that it requires the solution of a dense linear system of equa-

Manuscript received April 21, 2005; revised June 23, 2005

The authors are with the Department of Information Technology (INTEC), Ghent University, B-9000 Gent, Belgium (e-mail: Davy.Pissoort@intec.ugent. be).

Digital Object Identifier 10.1109/LAWP.2005.854000

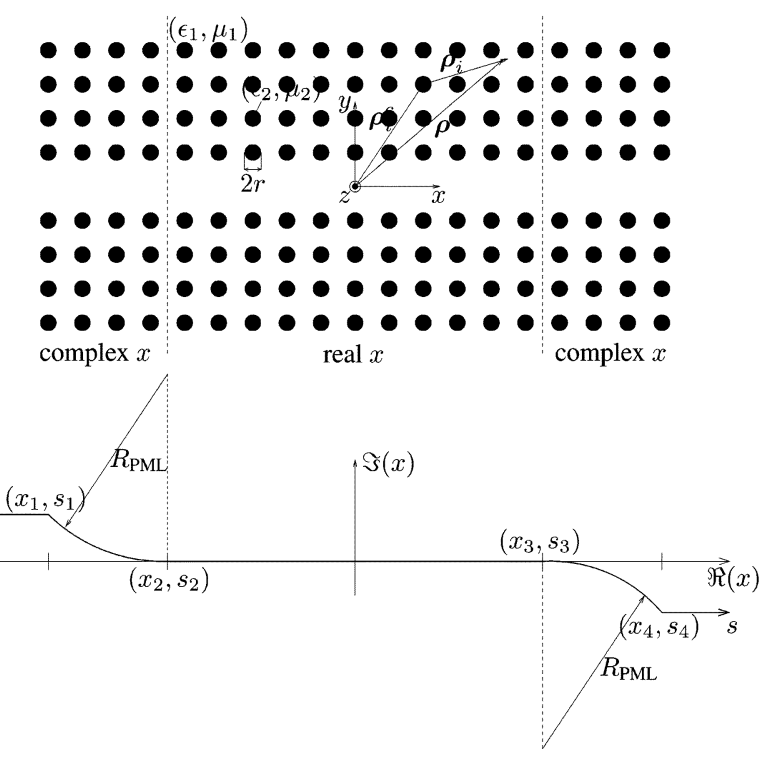

Fig. 1. Transformation coordinate system.

tions whose dimension is proportional to the number of cylinders in the EC device. Recently, there has been a considerable interest in the development of fast schemes to iteratively solve such systems [6]. A popular fast scheme is the multilevel fast multipole algorithm (MLFMA), which is the multilevel version of the fast multiple method (FMM). With this scheme, the cost per iteration scales nearly linearly with the number of cylinders. In this letter, the FMM is extended to the use of the PML-based absorbing boundary conditions.

\section{MultiPle SCATtering TeChNIQUE}

This section details the MST for characterizing 2-D EC devices comprising $N_{c}$ identical, $z$-invariant, homogeneous, dielectric/magnetic circular cylinders with radius $r$ and constitutive parameters $\left(\epsilon_{2}, \mu_{2}\right)$ embedded in a homogeneous background medium with constitutive parameters $\left(\epsilon_{1}, \mu_{1}\right)$ (Fig. 1). Let $E^{\mathrm{i}}(\boldsymbol{\rho}) \boldsymbol{u}_{z}$, with $\boldsymbol{\rho}=(\rho, \phi)$ a global position vector in the $x y$-plane, denote a known incident $\mathrm{TM}_{\mathrm{z}}$-polarized electric field generated by impressed sources in the absence of any cylinders. The difference between the total electric field $E^{\mathrm{t}}(\boldsymbol{\rho}) \boldsymbol{u}_{z}$, defined as the field observed in the presence of the cylinders, and the incident field is called the scattered field $E^{\mathrm{s}}(\boldsymbol{\rho}) \boldsymbol{u}_{z}$. To describe these electric fields, a set of equivalent $z$ directed electric currents $J^{j}(\boldsymbol{\rho}) \boldsymbol{u}_{z}$ are introduced on the surface $S_{j}$ of every cylinder $j, j=1, \ldots, N_{c}$. Let $E^{\mathrm{s}, j}(\boldsymbol{\rho}) \boldsymbol{u}_{z}$ denote the electric field generated by $J^{j}(\boldsymbol{\rho}) \boldsymbol{u}_{z}$ in an unbounded medium with parameters $\left(\epsilon_{1}, \mu_{1}\right)$. For dielectric/magnetic cylinders, appropriate 
boundary impedances $\mathcal{Z}^{i}$ can be proffered. The boundary condition on the surface $S_{i}$ of the $i$ th cylinder can then be cast as

$$
E^{\mathrm{i}}(\boldsymbol{\rho})+\sum_{j=1}^{N_{c}} E^{\mathrm{s}, j}(\boldsymbol{\rho})=\mathcal{Z}^{i} J^{i}(\boldsymbol{\rho}), \quad \text { for } \boldsymbol{\rho} \in S_{i} .
$$

To solve (1), the surface currents are expanded into a Fourier series $J^{j}(\boldsymbol{\rho})=\sum_{n=-\infty}^{+\infty}\left(I_{n}^{j} / 2 \pi r\right) e^{j n \phi_{j}} \delta\left(\rho_{j}-r\right)$ in a local cylindrical coordinate system $\boldsymbol{\rho}_{j}=\left(\rho_{j}, \phi_{j}\right)$ with respect to the center of the corresponding cylinder. Likewise, (1) can be reexpressed as

$$
\begin{aligned}
E^{\mathrm{i}}(\boldsymbol{\rho})+\sum_{j=1}^{N_{c}} E^{\mathrm{s}, j}(\boldsymbol{\rho})=\sum_{\substack{n=-\infty \\
\text { for } \boldsymbol{\rho} \in S_{i}, i=1, \ldots, N_{c}}}^{+\infty} \mathcal{Z}_{n}^{i} \frac{I_{n}^{i}}{2 \pi r} e^{j n \phi_{i}}
\end{aligned}
$$

with [7]

$$
\mathcal{Z}_{n}^{i}=\left[\frac{k_{2} J_{n}^{\prime}\left(k_{2} r_{2}\right)}{\mu_{2} J_{n}\left(k_{2} r_{2}\right)}-\frac{k_{1} J_{n}^{\prime}\left(k_{1} r_{1}\right)}{\mu_{1} J_{n}\left(k_{1} r_{1}\right)}\right]^{-1} .
$$

Here, $k_{\alpha}=\omega \sqrt{\epsilon_{\alpha} \mu_{\alpha}}$ is the wavenumber inside a medium with constitutive parameters $\left(\epsilon_{\alpha}, \mu_{\alpha}\right) ; J_{n}(\cdot)$ is the $n$ th-order Bessel function of the first kind and $H_{n}^{(2)}(\cdot)$ is the $n$ th-order Hankel function of the second kind. In practice, the range of the modal index $n$ can be restricted to $n=-K, \ldots, K$, with $K$ a small positive integer. The scattered electric field $E^{\mathrm{s}, j}(\boldsymbol{\rho})$ is given by

$E^{\mathrm{s}, j}(\boldsymbol{\rho})=-\frac{\omega \mu_{1}}{4} \int_{\boldsymbol{\rho}^{\prime} \in S_{j}} H_{0}^{(2)}\left(k_{1}\left|\boldsymbol{\rho}-\boldsymbol{\rho}^{\prime}\right|\right) J^{j}\left(\boldsymbol{\rho}^{\prime}\right) d \boldsymbol{\rho}^{\prime}$.

Inserting (4) into (2), truncating the infinite sums to a finite number, and testing the resulting equation with $T_{m}^{i}(\rho)=$ (1) $/(2 \pi r) e^{-j m \phi_{i}} \delta\left(\rho_{i}-r\right), m=-K, \ldots, K ; i=1, \ldots, N_{c}$, results in a linear system of equations

$\sum_{n=-K}^{K} \sum_{j=1}^{N_{c}} Z_{m n}^{i j} I_{n}^{j}=E_{m}^{i}, m=-K, \ldots, K, i=1, \ldots, N_{c}$.

All examples presented below involve ECs composed of dielectric cylinders with constitutive parameters $\left(\epsilon_{2}, \mu_{2}\right)=$ $\left(11.56 \epsilon_{0}, \mu_{0}\right)$ and radius $r=0.18 a$ that are arranged on a Cartesian lattice with a square unit cell and lattice constant a. All cylinders reside in free space, viz. $\left(\epsilon_{1}, \mu_{1}\right)=\left(\epsilon_{0}, \mu_{0}\right)$. This EC has a bandgap that extends from $k_{1}=0.604(\pi / a)$ to $k_{1}=0.886(\pi / a)$.

\section{FASt Multipole Method}

When solving system (5) iteratively, many matrix-vector multiplications are required. In the FMM, the computational complexity and memory requirements are minimized by dividing the $N_{c}$ cylinders into boxes, each of which contain about $M_{c}$ cylinders. The crux of the FMM is that it considers box-to-box interactions in stead of unknown-to-unknown interactions. As this idea of box-to-box interactions can only be applied if these two boxes are sufficiently separated, the matrix-vector multiplication is decomposed in two contributions, namely, the near-field and the far-field contribution, as follows:

$$
Z I=Z_{\mathrm{nf}} \boldsymbol{I}+Z_{\mathrm{ff}} \boldsymbol{I}
$$

As the matrix $Z_{\mathrm{nf}}$ is a sparse matrix with $O\left(N_{c}\right)$ nonzero elements, the computational complexity to calculate $\boldsymbol{Z}_{\mathrm{nf}} \boldsymbol{I}$ scales as $O\left(N_{c}\right)$. To see how $\boldsymbol{Z}_{\mathrm{ff}} \boldsymbol{I}$ can be computed more efficiently using box-to-box interactions, consider two sufficiently separated circular boxes with radius $R$. The source box is centered about $\rho_{s}^{c}$ and comprises $M_{s}$ cylinders centered about $\rho_{j}^{c}, j=1, \ldots, M_{s}$. Likewise, the observation box is centered about $\boldsymbol{\rho}_{o}^{c}$ and comprises $M_{o}$ cylinders centered about $\boldsymbol{\rho}_{i}^{c}, i=1, \ldots, M_{o}$. Let $I_{n}^{j}$ denote the $n$th modal unknown on cylinder $j$ inside the source box, $j=1, \ldots, M_{s} ; n=-K, \ldots, K$. Following a similar reasoning as in [6], it can be shown that the box-to-box interaction from the source to the observation box can be expressed as:

$$
\begin{aligned}
\sum_{j=1}^{M_{s}} \sum_{n=-K}^{K} Z_{m n}^{i j} I_{n}^{j} \approx & -\frac{\omega \mu_{1}}{4} \sum_{q=-Q}^{Q} J_{m}\left(k_{1} r\right) \\
& \times e^{-j\left[\boldsymbol{k}_{1}\left(\phi_{q}\right) \cdot\left(\boldsymbol{\rho}_{i}^{c}-\boldsymbol{\rho}_{o}^{c}\right)+m\left(\phi_{q}+\frac{\pi}{2}\right)\right]} \\
& \times T_{q}\left(k_{1}, \rho_{s o}^{c}, \phi_{s o}^{c}\right) \times \sum_{j=1}^{M_{s}} \sum_{n=-K}^{K} J_{n}\left(k_{1} r\right) \\
& \times e^{j\left[\boldsymbol{k}_{1}\left(\phi_{q}\right) \cdot\left(\boldsymbol{\rho}_{j}^{c}-\boldsymbol{\rho}_{s}^{c}\right)+n\left(\phi_{q}+\frac{\pi}{2}\right)\right]} I_{n}^{j}
\end{aligned}
$$

for $i=1, \ldots, M_{o}$ and $m=-K, \ldots, K$. Here, $\boldsymbol{k}_{1}(\phi)=$ $k_{1}(\cos \phi+\sin \phi), \rho_{s o}^{c}$ and $\phi_{s o}^{c}$ are the length and the angle with respect to the positive $x$-axis of the vector $\boldsymbol{\rho}_{s o}^{c}=\boldsymbol{\rho}_{o}^{c}-\boldsymbol{\rho}_{s}^{c}$ connecting the centers of the observation and the source box. The diagonal translation operator is defined as

$$
T_{q}(k, \rho, \phi)=\frac{1}{2 Q+1} \sum_{q^{\prime}=-Q}^{Q} H_{q^{\prime}}^{(2)}(k \rho) e^{j q^{\prime}\left(\phi-\phi_{q}-\frac{\pi}{2}\right)} .
$$

From (7), it is seen that the box-to-box interactions are calculated in three stages. First the radiation pattern of the source group is sampled into $2 Q+1$ outgoing plane waves in the directions $\phi_{q}=(2 \pi q / 2 Q+1), q=-Q, \ldots, Q$. Second, these plane waves are converted into $2 Q+1$ incoming plane waves for the observation box upon multiplication by the translation operator (8). Finally, the contribution of each plane wave is projected onto each cylinder in the observation box. According to the theory founded in [6], the number of samples should equal $2 Q+1=4 k_{1} R+C\left(k_{1} R\right)^{1 / 3}$. In practice, a certain accuracy is selected on beforehand. The appropriate sampling rate is found by simply increasing $Q$ until the desired accuracy is obtained.

In the MLFMA, the entire EC device is first enclosed in the smallest square box that can contain the EC (level 1). Then this box is divided into four square subboxes to form level 2. In its turn, each box of level 2 is divided into four square subboxes to generate the third level. This process is repeated until the finest level $L$, in which every box has a size of $0.2 \lambda-0.5 \lambda$, is reached. While performing matrix-vector multiplications, interactions between two boxes are calculated using the previously described FMM approach on the highest level on which these 


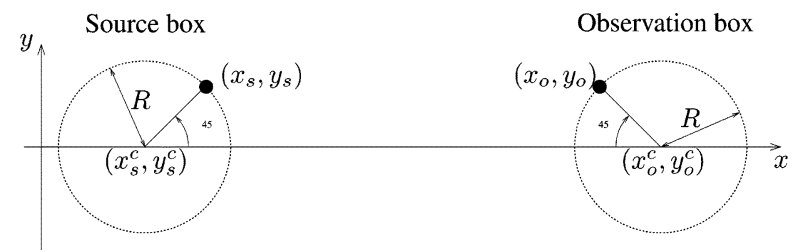

Fig. 2. Source and observation box for testing the accuracy, complex coordinates.

two boxes are well-separated. All the interactions that remain on the lowest level are computed in the classical way and constitute the near field contribution.

\section{COMPLEX COORDINATES}

Although the MST can only handle finite EC devices, it does not preclude the characterization of EC devices with semiinfinite waveguide attachments. Such characterization can be achieved by terminating sufficiently long EC device waveguide appendages by PML-based absorbing boundary conditions [1]. Because of the complex coordinate interpretation of a PML, this can be seen as if the EC waveguides make a bend into the complex plane. In [1], it was heuristically shown that a good absorption is obtained with a circular PML. This means that, for example, for an EC waveguide appendage in the positive $x$ direction, the $x$ coordinates of the cylinders in the extra periods become complex according to a circle with radius $R_{\mathrm{PML}}$

$$
x_{n}=x_{0}+R_{\mathrm{PML}} \sin (n \alpha)-j R_{\mathrm{PML}}[1-\cos (n \alpha)]
$$

with $\alpha=2 \arcsin (a) /\left(2 R_{\mathrm{PML}}\right)$ and $n=1, \ldots, N_{\mathrm{PML}}$. Here, $x_{n}$ is the $x$ coordinate of a cylinder in the $n$th period in the PML region; $x_{0}$ is the (real) $x$ coordinate of a cylinder in the last period that is not in the PML region. Typical values for the different parameters of the PML region are $R_{\mathrm{PML}}=10 a$ and $N_{\mathrm{PML}}=7$. This section will detail on how to incorporate these complex coordinates in the MLFMA framework. In [8], it is shown that the aforementioned addition theorem, which is the foundation of both the MST and the MLFMA, remains valid for complex coordinates.

First suppose that for the construction of the boxes and the MLFMA tree, only the real parts of the coordinates of the cylinders' centers are considered. In this case, a cylinder is inside a box if the real parts of its coordinates are located inside that box. It is obvious that also all box centers are real. Consider the configuration depicted in Fig. 2. It comprises a circular source and observation box with radius $R$. The distance between their centers is $\beta R$. Both boxes comprise one cylinder. The coordinates of the source and observation cylinder are

$$
\begin{aligned}
& \left(x_{s}, y_{s}\right)=\left(x_{s}^{c}+\frac{R}{\sqrt{2}}, y_{s}^{c}+\frac{R}{\sqrt{2}}\right) \\
& \left(x_{o}, y_{o}\right)=\left(x_{s}^{c}+\beta R-\frac{R}{\sqrt{2}}-j \alpha_{o} R, y_{s}\right) .
\end{aligned}
$$

Define the relative error $\delta$ as follows:

$$
\delta=\left|\frac{\left(Z_{m n}^{o s}\right)_{\mathrm{FMM}}-\left(Z_{m n}^{o s}\right)_{\mathrm{Class}}}{\left(Z_{m n}^{o s}\right)_{\mathrm{FMM}}}\right|
$$

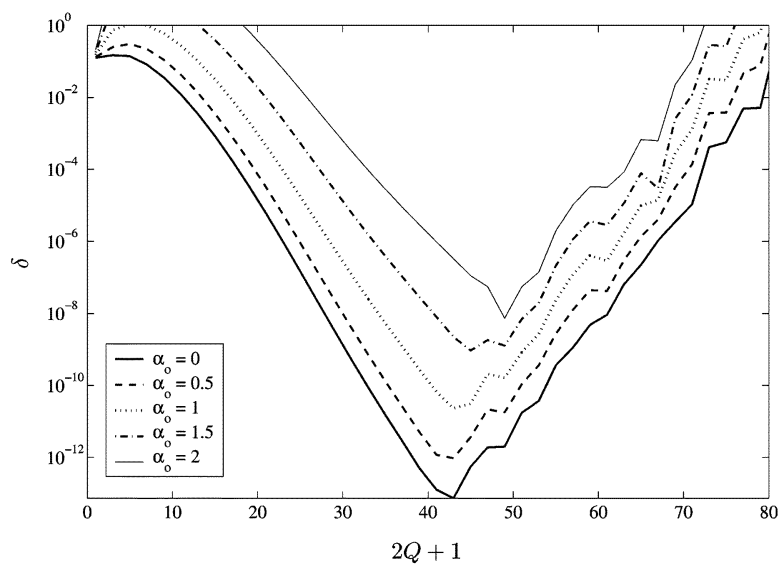

Fig. 3. Accuracy for $R=(\lambda / 2)$ and $\beta=6$, real box centers $\left(\alpha_{o}^{c}=0\right)$.

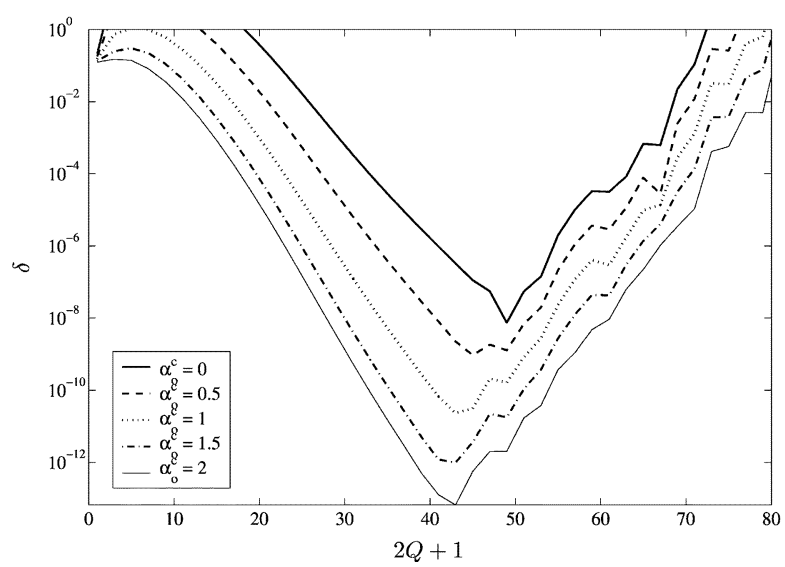

Fig. 4. Accuracy for $R=(\lambda / 2), \beta=6$, and $\alpha_{o}=2$, complex box centers.

Here, $\left(Z_{m n}^{o s}\right)_{\text {Class }}$ is the interaction between the $n$th modal unknown on the source cylinder and the $m$ th modal unknown on the observation cylinder calculated with (6); $\left(Z_{m n}^{o s}\right)_{\mathrm{FMM}}$ stands for the same interaction, but calculated using FMM. Fig. 3 shows the relative error for $m=n=0$ versus the sampling rate for increasing imaginary part of the $x$ coordinate of the observation cylinder with $k_{1}=0.72(\pi / a), R=(\lambda / 2)$, and $\beta=6$. The minimal achievable $\delta$ increases with increasing $\alpha_{o}$ and this minimum requires a higher $Q$. This yields some problems when choosing the optimal $Q$ on each level in the MLFMA. If only real coordinates are used for this choice, the required accuracy will not be obtained for interactions involving complex coordinates. When considering also complex coordinates, the sampling rate will be much higher than necessary for the interactions with only real coordinates. Also, it is clear that for very large imaginary parts the FMM will break down. To circumvent these difficulties, the coordinates of the boxes' centers will be allowed to be complex, too. For the above example, the coordinates of the source box remain real, but the coordinates of the observation box are $\left(x_{o}^{c}, y_{o}^{c}\right)=\left(x_{s}^{c}+\beta R-j \alpha_{o}^{c} R, y_{s}^{c}\right)$. Fig. 4 shows $\delta$ for $m=n=0$ versus the sampling rate for $\alpha_{o}=2$ and for increasing values of $\alpha_{o}^{c}$. A better accuracy can be achieved and this for a sampling rate that is the same as for real coordinates. When making the center of the box 


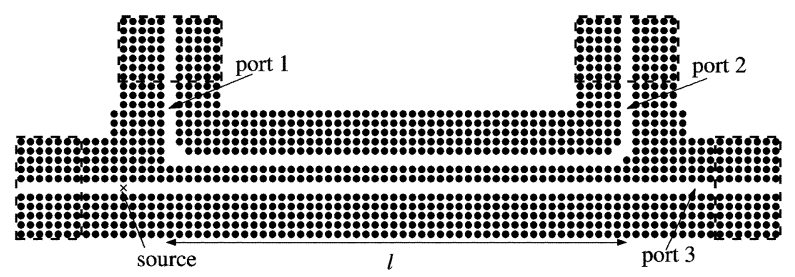

Fig. 5. EC waveguide coupler.

also complex the lost accuracy from making the center of the cylinders complex is fully recovered.

An issue that still remains to be solved is how to construct the boxes and the MLFMA tree when allowing complex coordinates for the box centers. Recall that the coordinates of the cylinders in the PML regions become complex according to a pre-described path, e.g., (9) for a circular PML in the positive $x$ direction. The coordinates of the box centers will be chosen such that they also follow this path. Consider, for example, the configuration depicted in Fig. 1. A straight EC waveguide parallel to the $x$ direction is sandwiched between two circular PML regions with radius $R_{\mathrm{PML}}$. In this figure, $\Re(\cdot)$ and $\Im(\cdot)$ denote the real and imaginary part, respectively. A new real coordinate $s$ is introduced. This coordinate follows the circles according to which the coordinates become complex, as indicated in Fig. 1. These circles stop at the last cylinders in the complex plane and from these points on the parameters $s$ follows a line parallel to the real $x$-axis. To construct the boxes and the MLFMA tree, the complex $x$ coordinates of all cylinders are first transformed into the real $s$ coordinates. The boxes are constructed based on these $s$ coordinates. The corresponding complex $x$ coordinates of the box centers can be easily calculated. The translation operators, the outgoing, and incoming plane waves are calculated using these complex coordinates.

\section{EXAMPLE}

The versatility of the adapted MLFMA is demonstrated here via its application to the characterization of the ultracompact two-channel multiplexer-demultiplexer depicted in Fig. 5, [9], [10]. The combination of two EC waveguides can support even and odd modes having different propagation constants $\beta_{e}$ and $\beta_{o}$, respectively. The normalized output power at ports 1,2 , and 3 can be estimated using the coupled mode theory [9]

$$
\begin{aligned}
& P_{1}=0 \\
& P_{2}=\sin ^{2}(\kappa l) \\
& P_{3}=\cos ^{2}(\kappa l)
\end{aligned}
$$

with $\kappa=(\pi / L)=\left(\left|\beta_{e}-\beta_{o}\right| / 2\right)$. Contrary to the multiple scattering technique, coupled mode theory cannot account for reflections from the $90^{\circ}$ waveguide bends. Fig. 6 shows the transmission spectra calculated with the coupled mode theory and the multiple scattering technique in combination with the MLFMA for a coupling length of $l=310 a$, assuming that $a=540 \mathrm{~nm}$. In the MLFMA, circular PML regions are added at the output ports. When comparing the results of both schemes, it is noted that the normalized output power calculated with the MLFMA is smaller than one. This is due to leakage from the sides of the EC

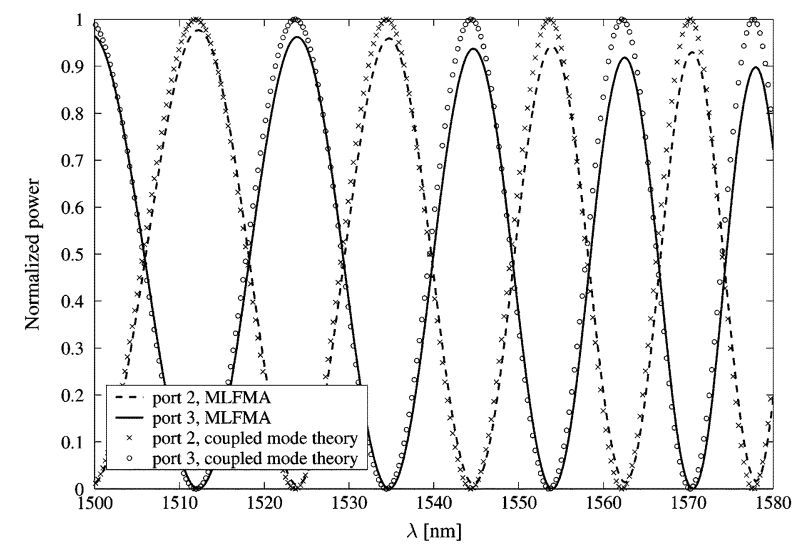

Fig. 6. Transmission spectrum of an EC coupler with $l=310 a$.

waveguides. This example comprises 10752 unknowns and one matrix-vector multiplication takes $0.3 \mathrm{~s}$. With a block- diagonal preconditioner, the BICGSTAB iterative solver requires about 200 iterations to solve the system of equations (5) to a tolerance of $10^{-4}$. This takes about $240 \mathrm{~s}$ for one frequency point. The memory requirements are $74 \mathrm{Mb}$.

\section{CONCLUSION}

The FMM has been extended to the use of complex coordinates to incorporate PML-based absorbing boundary conditions in integral equation like techniques. The centers of the boxes in the MLFMA tree are chosen along the predescribed path according to which the EC waveguides enter into the complex plane at the output ports of the EC device. This idea has been applied to the simulation of ultracompact two-channel multiplexer-demultiplexer.

\section{REFERENCES}

[1] D. Pissoort and F. Olyslager, "Termination of periodic waveguides by pmls in time-harmonic integral equation like techniques," IEEE Antennas Wireless Propag. Lett., vol. 2, no., pp. 281-284, 2003.

[2] G. Tayeb and D. Maystre, "Rigorous theoretical study of finite-size two-dimensional photonic crystals doped by microcavities," J. Opt. Soc. Amer. A, vol. 14, no. 12, pp. 3323-3332, Dec. 1997.

[3] J. D. Joannopoulos, R. D. Meade, and J. N. Winn, Photonic Crystals, Molding the Flow of Light. Princeton, NJ: Princeton Univ. Press, 1995.

[4] W. C. Chew and W. H. Weedon, "A 3D perfectly matched medium from modified Maxwell's equations in stretched coordinates," Microw. Opt. Technol. Lett., vol. 7, no. 13, pp. 599-604, Sep. 1994.

[5] W. C. Chew, J. M. Jin, and E. Michielssen, "Complex coordinate stretching as a generalized absorbing boundary condition," Microw. Opt. Technol. Lett., vol. 15, no. 6, pp. 363-369, Aug. 1997.

[6] W. C. Chew, J. M. Jin, E. Michielssen, and J. Song, Fast and Efficient Algorithms in Computational Electromagnetics. Norwood, MA: Artech House, 2001.

[7] D. Pissoort, B. Denecker, P. Bienstman, F. Olyslager, and D. De Zutter, "Comparative study of three methods for the simulation of two-dimensional photonic crystals," J. Opt. Soc. Amer. A, vol. 21, pp. 2186-2195, Nov. 2004.

[8] W. Magnus, F. Oberhettinger, and R. P. Soni, Formulas and Theorems for the Special Functions of Mathematical Physics. Berlin, Germany: Springer-Verlag, 1966.

[9] M. Koshiba, "Wavelength division multiplexing and demultiplexing with photonic crystal waveguide couplers," J. Lightwave Technol., vol. 19, no. 12, pp. 1970-1975, Dec. 2001.

[10] S. Boscolo, M. Midrio, and C. G. Someda, "Coupling and decoupling of electromagnetic waves in parallel 2D photonic crystal waveguides," IEEE J. Quantum Electron., vol. 1, no. 38, pp. 47-53, Jan. 2002. 